\title{
Long noncoding RNA TSLNC8 suppresses cell proliferation and metastasis and promotes cell apoptosis in human glioma
}

\author{
DONG $\mathrm{CHEN}^{1}$ and $\mathrm{XIN} \mathrm{YU}^{2}$ \\ ${ }^{1}$ Department of Neurosurgery, Tianjin Huanhu Hospital, Tianjin 300350; \\ ${ }^{2}$ Department of Surgery, Operating Room, Tianjin First Central Hospital, Tianjin 300192, P.R. China
}

Received August 13, 2017; Accepted August 1, 2018

DOI: $10.3892 / \mathrm{mmr} .2018 .9609$

\begin{abstract}
Glioma is among the most common primary brain tumors and one of the most aggressive and lethal forms of human cancer. Long noncoding RNAs (lncRNAs) have demonstrated great importance in the development and progression of cancer. The present study aimed to investigate the role of the novel tumor suppressive lncRNA on Chromosome 8p12 (TSLNC8), in cell proliferation, metastasis and apoptosis in human glioma. It was initially reported that the relative transcript levels of TSLNC8 were significantly decreased in human glioma tissues and cultured glioma cells, as evidenced by RT-qPCR. Among clinical variables, the expression of TSLNC8 was negatively associated with tumor size, distant metastasis, and tumor, node and metastasis stage. MTT assay demonstrated that overexpression of TSLNC8 in glioma cell lines U25-MG and SWO38 decreased, whereas knockdown of TSLNC8 in glioma cells SHG-44 and BT325 increased the cell proliferative rate over 5 consecutive days. Additionally, cell metastasis was inhibited in U251 and SWO38 cells when cells were transfected with TSLNC8-expressing plasmid as observed via Transwell and wound-healing assays. Furthermore, cell apoptotic rate was upregulated in TSLNC8 plasmid-treated U251 and SWO38 cells, and inhibited by siRNA against TSLNC8 in SHG-44 and BT325 cells by cell apoptotic assay. The relative activities of caspase- 3 and caspase- 9 were increased by TSLNC8 overexpression and decreased by TSLNC depletion; however, the activity of caspase- 8 remained unchanged. The results of the present study demonstrated the inhibitory effects of TSLNC8 in human glioma, which may contribute to advancement in the diagnosis and treatment of patients with glioma in clinic.
\end{abstract}

Correspondence to: $\mathrm{Dr} \mathrm{Xin} \mathrm{Yu}$, Department of Surgery, Operating Room, Tianjin First Central Hospital, 24 Fukang Road, Tianjin 300192, P.R. China

E-mail: xinyu8912@yeah.net

Key words: TSLNC8, glioma, proliferation, metastasis, apoptosis

\section{Introduction}

Glioma is the most common malignancy of brain tumor, accounting for $>32 \%$ of all brain tumors (1) and $~ 80 \%$ of malignancies in the brain (2). The overall median survival for patients with glioma is between 16 to 18 months $(3,4)$. Notable advances in the therapeutic strategies of glioma have been made in the last decade, including neurosurgical approaches, chemotherapy and radiotherapy; however, the prognosis of glioma remains poor, mainly due to the fact that glioma cells are highly aggressive and capable of infiltrating adjacent normal brain tissue, leading to the failure of complete dissection of the tumor by surgery in the brain $(5,6)$. Recurrence and resistance to chemotherapy are another two influential factors responsible for poor prognosis (7-9). Thus, developing novel strategies and investigating innovative therapeutic agents for patients diagnosed with glioma is imperative and urgently required.

Long noncoding RNAs (lncRNAs) are a class of RNAs that are non-protein coding and longer than 200 nucleotides in length (10-12). With the continuous advances of research approaches, lncRNAs are widely known and have recently undergone a rapid expansion in research and discovery. LncRNAs are closely associated with tumor development (13), particularly in the progression of glioma (14). For instance, IncRNA MEG63 contributes to cisplatin-induced apoptosis via the suppression of autophagy in human glioma (15). LncRNA CRNDE induces inflammation via the Toll-like receptor3-nuclear factor- $\kappa \mathrm{B}$-cytokine signaling pathway (16).

Tumor suppressive IncRNA on Chromosome $8 \mathrm{p} 12$ (TSLNC8) is a novel IncRNA, which is also named LINC00589 (17). TSLNC8 has been reported to be deleted and downregulated in human liver cancers. TSLNC8 serves its tumor suppressive role by physically interacting with transketolase and signal transducer and activator of transcription 3 (STAT3) and inactivation of the interleukin-6/STAT3 signaling pathway. In addition, TSLNC8 may inhibit the phosphorylation of STAT3, resulting in the low transcriptional activity of STAT3 in human liver cancer (17). Thus, TSLNC8 has been identified as a prognostic predictor for liver cancer patients in clinic; however, the role of TSLNC8 in human glioma requires further investigation.

In the present study, the transcript levels of TSLNC8 were observed to be significantly decreased in human glioma tissues 
compared with in noncancerous counterparts, and were negatively associated with tumor size, distant metastasis and tumor, node and metastasis (TNM) stage. Overexpression of TSLNC8 in glioma cells inhibited, while knockdown of TSLNC8 promoted cell proliferation and metastasis. TSLNC8 was also demonstrated to regulate cell apoptosis via the intrinsic pathway. The findings demonstrated the role of TSLNC8 in human glioma, which may contribute to the clinical diagnosis and treatment of the disease.

\section{Materials and methods}

Human samples. A total of 80 patients with diagnosed glioma (Male: Female, 59:21, age) were enrolled in the present study. For all patients, the dissected glioma tissues and their adjacent noncancerous tissues were collected from Tianjin First Center Hospital (Tianjin, China) during January 2014 to December 2016. All of the tissues were frozen in liquid nitrogen following dissection from patients during surgeries. Clinical data were also recorded for statistical analysis. Each patient provided informed consent to participate in the present study, which was approved by the Ethics Committee of Tianjin First Center Hospital.

Cell culture and transfection. Human glioma cell lines BE-2C and BT325 were purchased from the American Type Culture Collection (Manassas, VA, USA). Human glioma cell line SWO38 was a kind gift from Jinan University (Guangzhou, China) (18). Human glioma cell lines U251-MG, SHG-44 and CHG-5 were purchased from the Cell Bank of Type Culture Collection of Chinese Academy of Science (Shanghai, China). Human normal neuronal cell line. Human normal astrocyte cells were purchased from ScienCell Research Laboratories, Inc. (San Diego, CA, USA; catalog no. 1830) and used as a normal control of glioma cell. All culture media (Dulbecco's modified Eagle's medium (DMEM; Gibco; Thermo Fisher Scientific, Inc., Waltham, MA, USA) were supplied with $10 \%$ fetal bovine serum (FBS, Gibco; Thermo Fisher Scientific, Inc.). Cells were maintained at $37^{\circ} \mathrm{C}$ in a humidified atmosphere containing $5 \% \mathrm{CO}_{2}$. Small interfering (si)RNA against TSLNC8 was designed and synthesized by Shanghai Shenggong Biology Engineering Technology Service, Ltd., (Shanghai, China) with the sequence of 5'-GCACATGAA CACATTGAAA-3' and the control siRNA sequence was 5'-GCAAAGTACACGTTACAAA-3' with the same source as the specific siRNAs. TSLNC8 expressing plasmid was constructed by our own lab with XhoI and HindIII restriction enzyme and cloned into pcDNA 3.1 vector. A total of $2 \mu \mathrm{g}$ siRNAs or expressing plasmid was transfected into cells with Lipofectamine ${ }^{\circledR} 2000$ (Invitrogen; Thermo Fisher Scientific, Inc.) according to the manufacturer's protocol. After $12 \mathrm{~h}$, the cell culture medium was replaced with new one. In all conditions, the culture medium was replaced every 2 days, unless otherwise stated.

$R N A$ isolation and reverse transcription-quantitative polymerase chain reaction ( $R T-q P C R)$. Total RNA from human tissues and all cultured cells was extracted using TRIzol ${ }^{\circledR}$ reagent (Thermo Fisher Scientific, Inc.). RNA was quantified by Nanodrop 2000 (Thermo Fisher Scientific, Inc.,
Wilmington, DE, USA) by measuring the optical density at a wavelength of $260 \mathrm{~nm}$. RT was then immediately performed using Prime Script TM Master Mix (Takara Bio, Inc., Otsu, Japan) according to the manufacturer's protocol. Subsequently, RT-qPCR was performed with SYBR ${ }^{\circledR}$ Premix EX Taq TM II (Takara Bio, Inc.) on the real-time PCR detection system ABI7900 (Applied Biosystems; Thermo Fisher Scientific, Inc.). GAPDH was used as the internal reference, and gene mRNA expression levels were calculated using the $2^{-\Delta \Delta C q}$ method (19). The thermocycling protocol was conducted as follows: Initial denaturation at $95^{\circ} \mathrm{C}$ for $5 \mathrm{~min}$, followed by 45 cycles of a three-step cycling program consisting of $10 \mathrm{sec}$ at $95^{\circ} \mathrm{C}$ (denaturation), $10 \mathrm{sec}$ at $60^{\circ} \mathrm{C}$ (primer annealing) and $10 \mathrm{sec}$ at $72^{\circ} \mathrm{C}$ (elongation), and a final extension step for $10 \mathrm{~min}$ at $72^{\circ} \mathrm{C}$. The primer sequences used for qPCR were as follows: TSLNC8 forward, 5'-TGATCCTCATAGTAT AATG-3' and reverse, 5'-AGTTCTTTAGCAGTACATG-3'; GAPDH forward, 5'-GTGGACATCCGCAAAGAC-3' and reverse, 5'-AAAGGGTGTAACGCAACTA-3'.

Cell proliferation analysis. Cell viability was determined via an MTT assay. A total of 1,000 U251-MG and SWO38 cells were transfected with TSLNC8-overexpressing plasmid and SHG-44 and BT325 cells were treated with siRNAs with or without TSLNC8 knockdown for $48 \mathrm{~h}$, trypsinized and reseeded in triplicate in 96-well plates at an initial density of 4,000 cells per well. Cell numbers were monitored for a total of 5 consecutive days. At each indicated time points (days 1,2, 3, 4 and 5), cell cultures were incubated with $10 \mu \mathrm{l}$ MTT solution $(5 \mathrm{mg} / \mathrm{ml}$, Beyotime Institute of Biotechnology, Haimen, China) per well for $2 \mathrm{~h}$ at room temperature. The absorbance was recorded at a wavelength of $570 \mathrm{~nm}$. Cell viability was defined as the cell number ratio of experimental groups to control cells.

Transwell assay. For cell migration assays, U251-MG and SWO38 cells were transfected with TSLNC8-overexpressing plasmid for $48 \mathrm{~h}$ and then trypsinized and collected by low-speed centrifugation $\left(1,000 \times \mathrm{g}, 4^{\circ} \mathrm{C}\right.$ for $\left.5 \mathrm{~min}\right)$ with serum-free DMEM. A total of $1 \times 10^{4}$ cells $(\sim 150 \mu 1)$ were spread into the upper chamber. The lower chamber was filled with $600 \mu$ DMEM supplemented with 10\% FBS. Subsequently, the plate was incubated for $24 \mathrm{~h}$ at $37^{\circ} \mathrm{C}$ in an incubator and the cells were allowed to grow freely. At $24 \mathrm{~h}$ post-seeding, the membrane was fixed with pre-cooled methanol at room temperature for $10 \mathrm{~min}$ and stained with crystal violet (1\%) for $5 \mathrm{~min}$ at room temperature. Cell migration was assessed by counting the cells that had migrated through the membrane. A total of 5 random fields were selected and images captured under a Nikon light microscope (Nikon Corporation, Tokyo, Japan) at a magnification of x100. For cell invasion assays, the membrane was pre-coated with Matrigel (Corning Incorporated, Corning, NY, USA) for $6 \mathrm{~h}$ in a $37^{\circ} \mathrm{C}$ incubator.

Wound-healing assay. U251-MG and SWO38 cells were transfected with TSLNC8-overexpressing plasmid for $48 \mathrm{~h}$ and were then cultured in DMEM in a 6-well culture plate at a density of $5 \times 10^{5}$ cells/well overnight until $90 \%$ confluence was attained. The culture medium was replaced with serum-free DMEM. A line was scratched in the single cell layer using a 
Table I. Association of TSLNC8 with clinical variables among 80 glioma patients.

\begin{tabular}{|c|c|c|c|c|c|}
\hline \multirow[b]{2}{*}{ Variable } & \multirow[b]{2}{*}{$\mathrm{N}$} & \multicolumn{2}{|c|}{ Expression of TSLNC8 } & \multirow[b]{2}{*}{ P-value } & \multirow[b]{2}{*}{ Coefficient $\left(\mathrm{R}^{2}\right)$} \\
\hline & & $\operatorname{Low}^{\mathrm{d}}(\mathrm{n}=43)$ & $\operatorname{High}^{\mathrm{d}}(\mathrm{n}=37)$ & & \\
\hline Age (years) & & & & 0.245 & -0.132 \\
\hline$<40$ & 16 & 6 & 10 & & \\
\hline $40-50$ & 24 & 14 & 10 & & \\
\hline$>50$ & 40 & 23 & 17 & & \\
\hline Sex & & & & 0.960 & -0.006 \\
\hline Male & 43 & 23 & 20 & & \\
\hline Female & 37 & 20 & 17 & & \\
\hline Tumor size $(\mathrm{T})$ & & & & $0.001^{\mathrm{c}}$ & -0.373 \\
\hline $\mathrm{T} 1$ and $\mathrm{T} 2^{\mathrm{a}}$ & 38 & 13 & 25 & & \\
\hline $\mathrm{T} 3$ and $\mathrm{T} 4^{\mathrm{b}}$ & 42 & 30 & 12 & & \\
\hline Lymph node metastasis $(\mathrm{N})$ & & & & 0.155 & -0.160 \\
\hline No & 28 & 12 & 16 & & \\
\hline N1 or above & 52 & 31 & 21 & & \\
\hline Distant metastasis (M) & & & & $0.004^{\mathrm{c}}$ & -0.317 \\
\hline M0 & 32 & 11 & 21 & & \\
\hline M1 & 48 & 32 & 16 & & \\
\hline TNM stage & & & & $0.003^{\mathrm{c}}$ & -0.323 \\
\hline $\mathrm{I} / \mathrm{II}$ & 24 & 7 & 17 & & \\
\hline III/IV & 56 & 36 & 20 & & \\
\hline
\end{tabular}

${ }^{\mathrm{a}} \mathrm{T} 1$ and $\mathrm{T} 2$ denotes tumor size $\leq 4 \mathrm{~cm} ;{ }^{\mathrm{b}} \mathrm{T} 3$ and $\mathrm{T} 4$ denotes tumor size $>4 \mathrm{~cm}$ or any size with distant metastasis; ${ }^{\mathrm{c}}$ denotes a negative association; ${ }^{\mathrm{d}}$ Low corresponds to lower expression than the median and high corresponds to higher expression than the median. TNM, tumor, mode and metastasis; TSLNC8, tumor suppressive lncRNA on Chromosome 8p12.

$10 \mu l$ pipette tip and the cells were then washed with PBS three times. Following incubation at $37^{\circ} \mathrm{C}$ for $24 \mathrm{~h}$, images of the migrated cells were observed and images were captured using a Nikon light microscope (magnification, x200).

Cell apoptosis. The Annexin V-fluorescein isothiocyanate (FITC)/propidium iodide (PI) assay was performed according to the manufacturer's protocol (Invitrogen; Thermo Fisher Scientific, Inc.). Briefly, a total of 10,000 U251-MG and SWO38 cells were plated into 6-well plates and transfected with TSLNC8-overexpressing plasmid for $48 \mathrm{~h}$; SHG-44 and BT325 cells were transfected with control or specific siRNA against TSLNC8. Subsequently, cells were washed with pre-cooled PBS, trypsinized, and re-suspended in $100 \mu \mathrm{l}$ of binding buffer with $2.5 \mu$ l FITC-conjugated Annexin V and $1 \mu \mathrm{l} \mathrm{PI}(100 \mu \mathrm{g} / \mathrm{ml})$. Cells were then incubated at room temperature for $15 \mathrm{~min}$ in darkness. A total of $\geq 10,000$ cells were collected and analyzed.

Relative caspase activities. The activities of caspase- $3,-8$ and -9 were determined using the caspase activity kits (Beyotime Institute of Biotechnology, Nantong, China) according to the manufacturer's protocols. Briefly, U251-MG and SWO38 cells were plated into 6 -well plates and transfected with TSLNC8-overexpressing plasmid for $48 \mathrm{~h}$; SHG-44 and BT325 cells were transfected with control or specific siRNA against
TSLNC8. Subsequently, cell lysates were collected by low speed centrifugation $\left(1,000 \mathrm{xg}\right.$, for $5 \mathrm{~min}$ at $\left.4^{\circ} \mathrm{C}\right)$. A total of $10 \mu \mathrm{l}$ protein from each sample were added into 96 -well plates and mixed with an aliquot of $80 \mu 1$ reaction buffer supplied with caspase substrates $(2 \mathrm{mM})$. Following incubation at $37^{\circ} \mathrm{C}$ for $4 \mathrm{~h}$, caspase activities were determined with a Tecan reader (Tecan Group, Ltd, Mannedorf, Switzerland) at an absorbance of $450 \mathrm{~nm}$.

Statistical analysis. GraphPad Prism version 5.0 (GraphPad Software, La Jolla, CA, USA) software was used for statistical analysis. Data are presented as the mean \pm standard deviation. The two-tailed Student's t-test was used to compare means of two groups, while one-way analysis of variance was used for comparisons among multiple groups ( $\geq 3$ groups), followed by a Least Significant Difference post hoc test. The Spearman correlation analysis was used to evaluate the experimental data in Table I. $\mathrm{P}<0.05$ was considered to indicate a statistically significant difference. Each experiment was repeated at least three times.

\section{Results}

LncRNA TSLNC8 is downregulated in human glioma in vivo and in vitro. First, the relative transcript levels of TSLNC8 in 80 glioma patients were analyzed in the present study. As 
presented in Fig. 1A, the expression of TSLNC8 was significantly decreased in $95 \%$ cases of glioma patients (76 cases) compared with in adjacent noncancerous tissues $(\mathrm{P}<0.01)$. Of note, TSLNC8 transcript levels higher than the median level were characterized as high TSLNC8 expression $(n=37)$; transcript levels lower than the median level were characterized as low TSLNC8 expression $(n=43)$. The clinical data were also analyzed and presented in Table I, which indicated that the relative transcript levels of TSLNC8 were negatively associated with tumor size $(\mathrm{P}=0.001)$, distant metastasis $(\mathrm{P}=0.004)$ and TNM stage $(\mathrm{P}=0.003)$, and not associated with age $(\mathrm{P}=0.2445)$, sex $(\mathrm{P}=0.960)$ and lymph node metastasis $(\mathrm{P}=0.155)$. Then, five glioma cell lines, including U251-MG, SHG-44, BT325, SWO38, CHG-5 and normal astrocyte cell line used as a control were analyzed. RT-qPCR analysis revealed that all of the five glioma cell lines exhibited significantly lower transcript levels of TSLNC8 compared with in the control astrocyte cells (Fig. 1B), of which U251-MG and SWO38 demonstrated the lowest expression levels of TSLNC8. Additionally, U251-MG and SWO38 cells exhibited the highest potential to migrate; SHG-44 and BT325 revealed the highest expression of TSLNC8 of the 7 glioma cell lines and the migration capacities of these cell lines were the lowest (Fig. 1B). Therefore, U251-MG and SWO38 were selected for the knockdown experiments and SHG-44 and BT325 were used for overexpression studies. All of these data showed that the transcript level of TSLNC8 was decreased in human glioma in vivo and in vitro.

Transcript levels of TSLNC8 are negatively associated with cell proliferation rate in human glioma. To investigate the role of TSLNC8 in human glioma, TSLNC8 was overexpressed in U251-MG and SWO38 cells and downregulated in SHG-44 and BT325 cells using an overexpression plasmid or siRNAs, respectively. When U251-MG and SWO38 cells were transfected with TSLNC8-overexpression plasmid, the transcript levels of TSLNC8 were significantly increased by 4.5 - and 4-fold, respectively (Fig. 2A). In addition, the expression levels of TSLNC8 in SHG-44 and BT325 cells were significantly decreased by $>50 \%$ of the original levels upon transfection with siTSLNC8 (Fig. 2B). Subsequently, the proliferation rate of the 4 glioma cell lines was analyzed. No significant alterations in the first 2 days in experimental cells were observed; however, cell proliferation decreased by $17 \%$ on day 4 and $19 \%$ on day 5 of U251 cells transfected with TSLNC8-expressing plasmid (Fig. 2C). Furthermore, SWO38 cell proliferation rate decreased on days 4 and 5 with TSLNC8 overexpression (Fig. 2D); the cell proliferation rate increased on days 4 and 5 in SHG-44 and BT325 cells transfected with siRNA against TSLNC8 (Fig. 2E and F, respectively). These results suggested that TSLNC8 may suppress cell proliferation in human glioma cells.

Transcript levels of TSLNC8 are negatively associated with cell metastasis in human glioma. As well as cell proliferation rate, the effects of TSLNC8 on cell metastasis were investigated. To this end, TSLNC8-expressing plasmid was transfected into U251-MG and SWO38 cells for $48 \mathrm{~h}$. Transwell assays revealed that $>400 \mathrm{U} 251$ and SWO38 cells were observed to migrate via the membrane; however, only 200 cells were counted
A

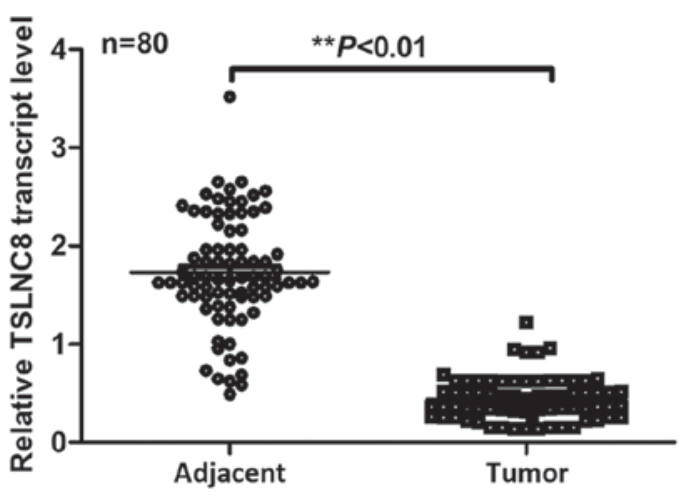

B

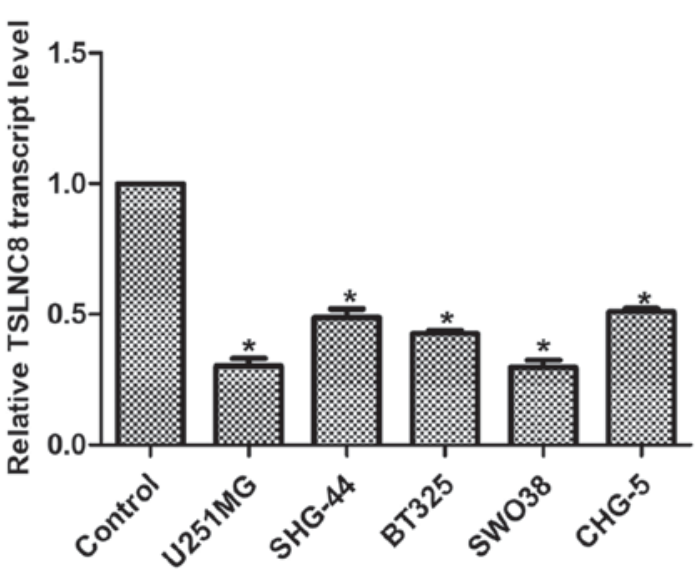

Figure 1. Long noncoding RNA TSLNC8 is downregulated in human glioma in vivo and in vitro. (A) RT-qPCR analysis were performed in tumor tissues and their adjacent noncancerous tissues from 80 clinical glioma patients. ${ }^{* *} \mathrm{P}<0.01$. (B) RT-qPCR analysis were performed in glioma cell lines (U251-MG, SHG-44, BT325, SWO38, and CHG-5) and a normal astrocyte cell line, which served as the control. ${ }^{*} \mathrm{P}<0.05$ vs. control. RT-qPCR, reverse transcription-quantitative polymerase chain reaction; TSLNC8, tumor suppressive lncRNA on Chromosome 8p12.

on the lower side of the membrane upon transfection with TSLNC8-expressing plasmid (Fig. 3A and B). Additionally, cell invasion was also inhibited by TSLNC8 overexpression in both U251-MG and SWO38 cells (Fig. 3A and C). Subsequently, wound-healing assays were also performed in U251-MG and SWO38 cells. As presented in Fig. 3D and E, the wound closure ability of U251-MG cells was inhibited by $>50 \%$ when cells were treated with TSLNC8-overexpression plasmid. Furthermore, the wound closure ability of SWO38 cells was also inhibited by $50 \%$ upon TSLNC8 overexpression (Fig. 3D and F). These data suggested that TSLNC8 suppressed cell metastasis in human glioma.

Transcript levels of TSLNC8 are positively associated with cell apoptosis in human glioma. Finally, cell apoptosis was assessed in glioma cells. As presented in Fig. 4A, cell apoptosis was increased by 2.7-fold in U251-MG cells and 2.5-fold in SWO38 cells of the TSLNC8 treated groups compared with in the control cells; the cell apoptotic rate of SHG-44 and BT325 cells were significantly decreased by $>50 \%$ upon siTSLNC8 stimulation (Fig. 4B). Subsequently, the relative caspase activities were analyzed. Treatment with TSLNC8-expressing plasmid of U251-MG and SWO38 cells 
A

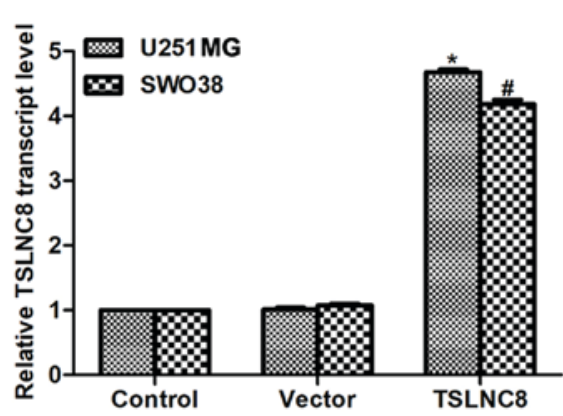

C

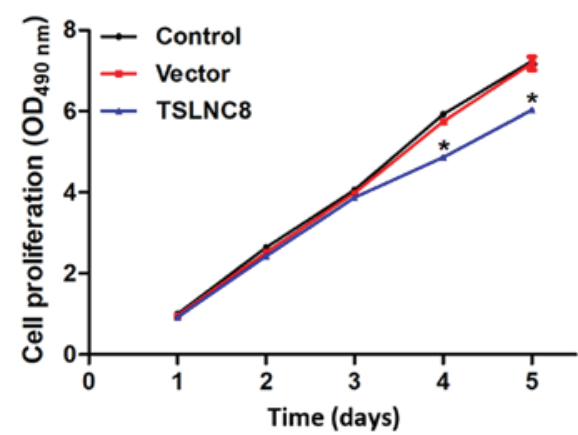

E

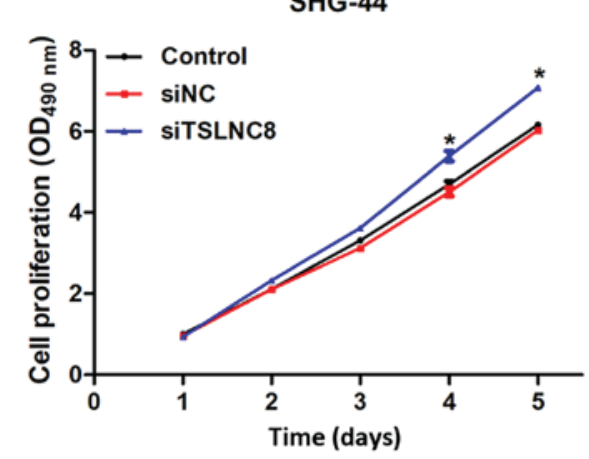

B

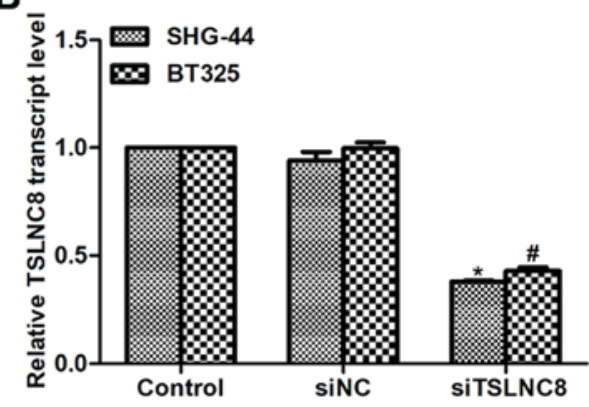

D

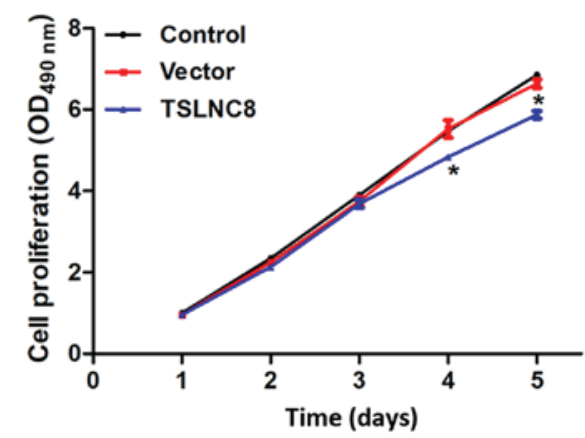

$\mathbf{F}$

BT325

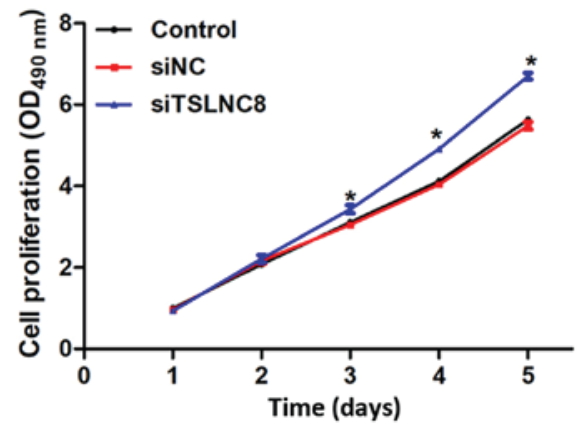

Figure 2. Transcript levels of TSLNC8 are negatively associated with cell proliferative rate in human glioma. (A) Transcript levels of TSLNC8 were examined in U251 and SWO38 cells upon TSLNC8 overexpression. ${ }^{*} \mathrm{P}<0.05$ vs. U251 control cells. ${ }^{~} \mathrm{P}<0.05$ vs. SWO38 control. (B) Transcript levels of TSLNC8 were examined in SHG-44 and BT325 cells upon TSLNC8 knockdown. ${ }^{*} \mathrm{P}<0.05$ vs. SHG-44 control cells. ${ }^{~} \mathrm{P}<0.05$ vs. BT325 control cells. (C) Cell proliferation rate was investigated in U251 cells transfected with TSLNC8 expressing plasmid for 5 consecutive days. (D) Cell proliferative rate was investigated in SWO38 cells transfected with TSLNC8-expressing plasmid for 5 consecutive five days. (E) Cell proliferative rate was investigated in SHG-44 cells transfected with siTSLNC8 for 5 consecutive days. (F) Cell proliferative rate was investigated in BT325 cells transfected with siTSLNC8 for 5 consecutive days. ${ }^{*} \mathrm{P}<0.05$ vs. Control. TSLNC8, tumor suppressive lncRNA on Chromosome 8p12; siTSLNC8, small interfering RNA against TSLNC8; siNC, negative control; OD, optical density.

increased, while stimulation of siTSLNC8 in SHG-44 and BT325 cells decreased the activities of caspase-3, respectively (Fig. 4C and D). Interestingly, the activity of caspase-8 remained stable upon transfection with TSLNC8-expressing plasmid or siTSLNC8 in glioma cells (Fig. 4E and F). The relative activities of caspase- 9 were also significantly altered, following similar trends to those exhibited by caspase-3, in the different glioma cell lines (Fig. 4G and H). As caspase-3 and -9 are key factors involved in the intrinsic apoptosis pathway and caspase- 8 serves significant roles in the extrinsic apoptotic pathway (20), the findings of the present study suggested that TSLNC8 may promote cell apoptosis via the intrinsic pathway in human glioma.

\section{Discussion}

Deletion in the short arm of chromosome 8 is among the most common genetic events in a variety of cancers, and deletions include Rho guanosine 5 '-triphosphate-ase activating protein (8p22) (21), leucine zipper tumor suppress 1 (8p21) (22) and tumor necrosis factor superfamily member 10c (8p21) (23). The present study demonstrated that a novel lncRNA TSLNC8, also located at 8p12, may serve a significant role in human glioma, which was consistent with the former findings of Zhang et al (17). The results of the present study suggested that TSLNC8 was downregulated in human glioma, which was associated with increased cell proliferative rate and cell 
A
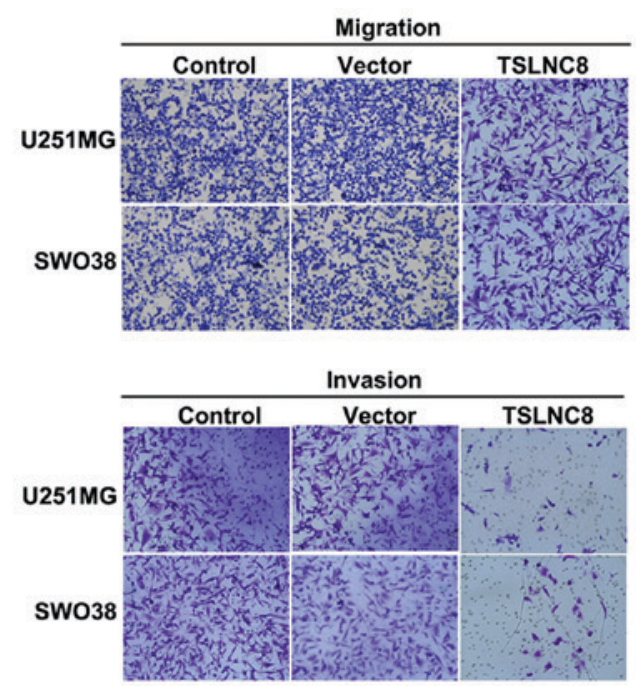

D

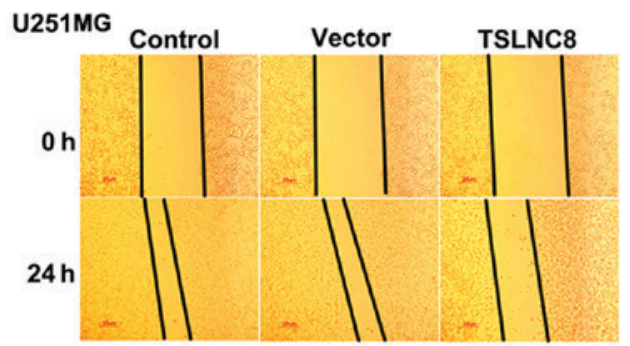

SW038

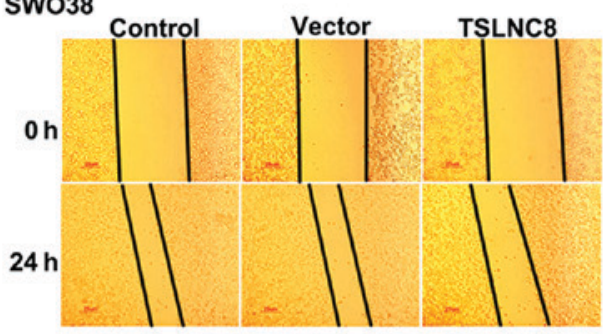

B

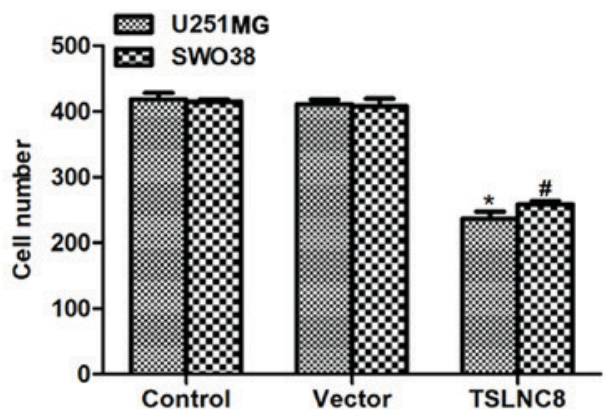

C

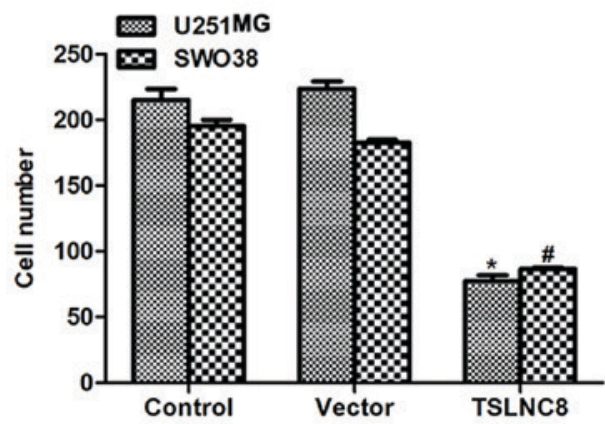

E

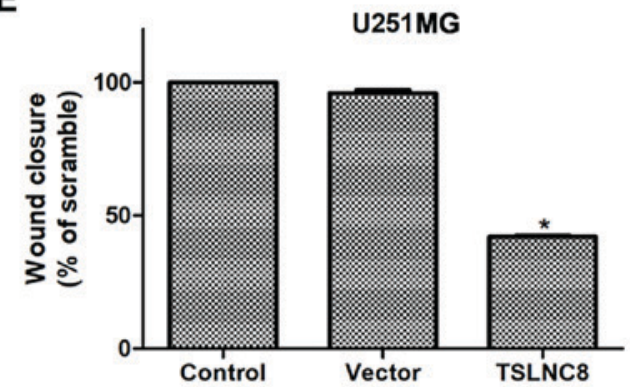

F

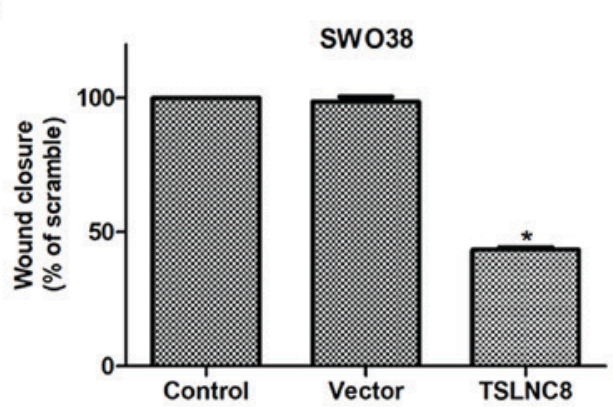

Figure 3. Transcript levels of TSLNC8 are negatively associated with cell metastasis in human glioma. (A) Representative images of Transwell assays in U251-MG and SWO38 cells in the presence and absence of TSLNC8 overexpression. Magnification, x200. (B) Quantification of cell migration assays of U251-MG and SWO38 cells in the presence and absence of TSLNC8 overexpression. (C) Quantification of cell invasion assays of U251-MG and SWO38 cells in the presence and absence of TSLNC8 overexpression. " $\mathrm{P}<0.05$ vs. U251-MG control cells. "P<0.05 vs. SWO38 control cells. (D) Representative images of wound-healing assays in U251-MG and SWO38 cells in the presence and absence of TSLNC8 overexpression. (E) Quantification of wound healing assays of U251-MG cells in the presence and absence of TSLNC8 overexpression. (F) Quantification of wound healing assays of SWO38 cells in the presence and absence of TSLNC8 overexpression. " P<0.05 vs. Control. TSLNC8, tumor suppressive lncRNA on Chromosome 8p12.

metastasis, as well as the decreased cell apoptotic capacity in human glioma cell lines; TSLNC8 may be a potential therapeutic target for cancer patients in clinic.

Cell proliferation and cell metastasis are two primary manifestations in the majority of malignancies $(24,25)$. Initiation of metastasis requires invasion, which was examined in the present study via Transwell and wound-healing assays. Invasion is enabled by epithelial-mesenchymal transition (EMT) $(26,27)$. Cells from primary tumors lose cell-cell adhesion mediated by E-cadherin repression and break through the basement membrane with increased invasive capacity, thus enter the bloodstream via intravasation (28). At novel metastatic sites, tumor cells undergo mesenchymal-epithelial transition by overexpressing $\mathrm{N}$-cadherin (28); the protein levels of E-cadherin and $\mathrm{N}$-cadherin require further investigation in future studies, as well as the detailed mechanism of the regulatory effects of TSLNC8 on human glioma. 
A

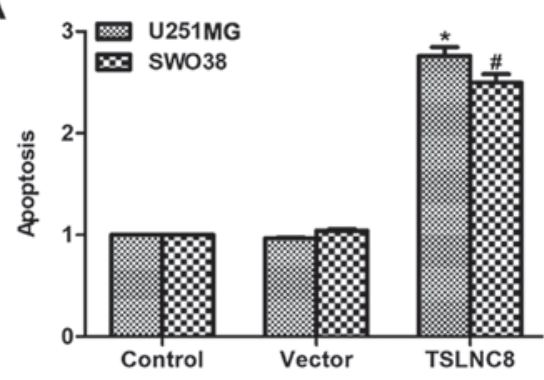

C

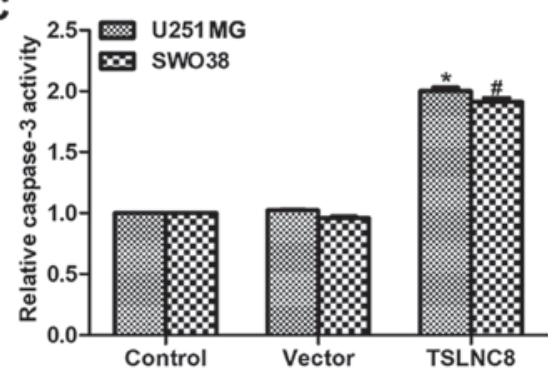

E

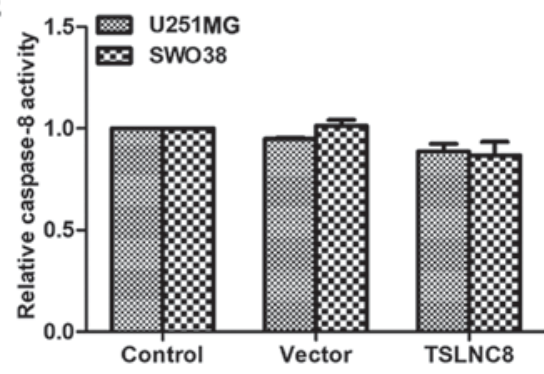

G

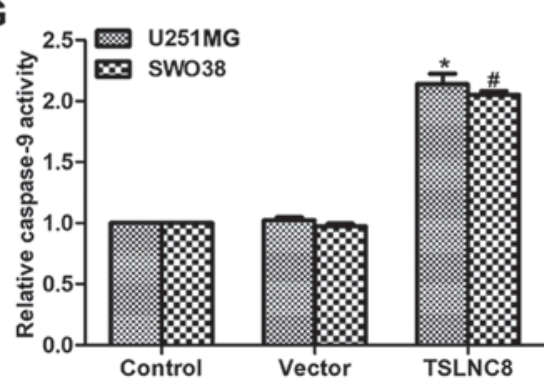

B

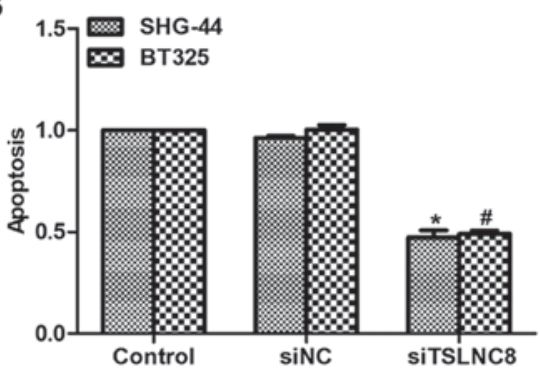

D

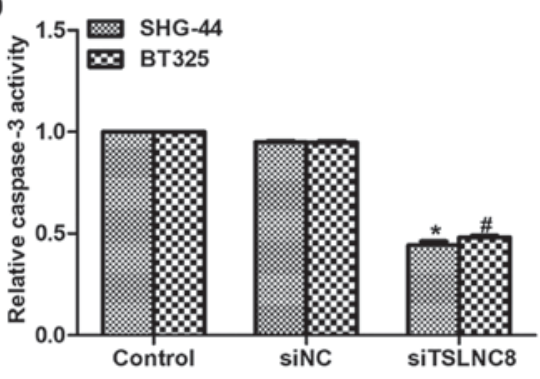

$\mathbf{F}$

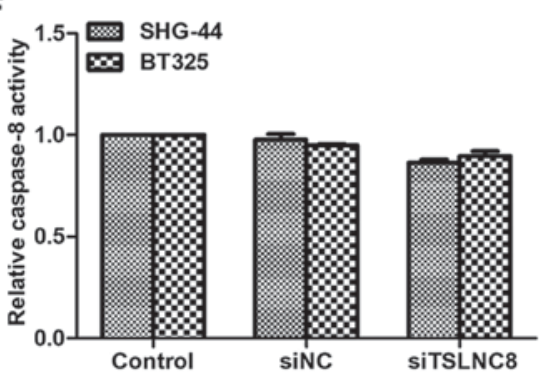

H

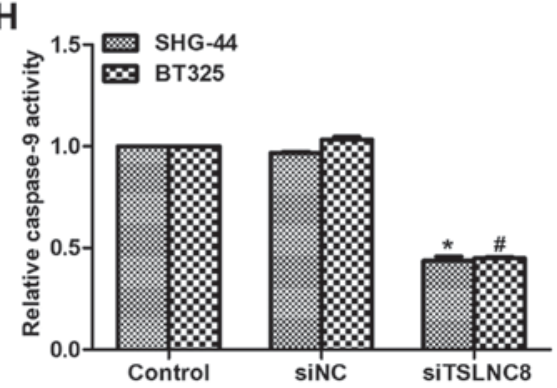

Figure 4. Transcript levels of TSLNC8 are positively associated with cell apoptosis in human glioma. (A) Cell apoptotic rate was assessed in U251-MG and SWO38 cells treated with TSLNC8-expressing plasmid. "P<0.05 vs. U251-MG control cells. " $\mathrm{P}<0.05$ vs. SWO38 control cells. (B) Cell apoptotic rate was assessed in SHG-44 and BT325 cells treated with TSLNC8 siRNA. "P<0.05 vs. in SHG-44 control cells. " $\mathrm{P}<0.05$ vs. BT325 control cells. (C) Relative activity of caspase-3 detected in U251-MG and SWO38 cells transfected with TSLNC8-expressing plasmid. " $\mathrm{P}<0.05$ vs. U251 control cells. ${ }^{\text {P }}<<0.05$ vs. SWO38 control cells. (D) Relative activity of caspase-3 detected in SHG-44 and BT325 cells transfected with TSLNC8 siRNA. "P<0.05 vs. SHG-44 control cells. ${ }^{\text {"P }}<0.05$ vs. BT325 control cells. (E) Relative activity of caspase-8 detected in U251-MG and SWO38 cells transfected with TSLNC8-expressing plasmid. (F) Relative activity of caspase-8 detected in SHG-44 and BT325 cells transfected with TSLNC8 siRNA. (G) Relative activity of caspase-9 detected in U251-MG and SWO38 cells transfected with TSLNC8-expressing plasmid. " $\mathrm{P}<0.05$ vs. U251-MG control cells. ${ }^{\#} \mathrm{P}<0.05$ vs. SWO38 control cells. (H) Relative activity of caspase-9 detected in SHG-44 and BT325 cells transfected with TSLNC8 siRNA. "P $<0.05$ vs. SHG-44 control cells. "P $<0.05$ vs. BT325 control cells. TSLNC8, tumor suppressive lncRNA on Chromosome 8p12; siTSLNC8, small interfering RNA against TSLNC8; siNC, negative control.

Numerous factors are involved in EMT, including programmed death-ligand 1 , twist-related protein 1 and 2 , and transforming growth factor $\beta 1$ (TGF $\beta 1$ ). TGF $\beta 1$ may promote tumor invasion and evasion of immune surveillance at the advanced stage of malignancies (29). EMT is favored and apoptosis is suppressed when TGF $\beta 1$ acts on activated Ras-expressing mammary epithelial cells, which may be reversed by the inducers of epithelial differentiation (30). As the inhibition of cell apoptosis is a good basis for tumor cell growth, the role of TSLNC8 in cell apoptosis was analyzed in the present study. TSLNC8 was observed to promote cell apoptosis by increasing the activities of caspase-3 and caspase- 9 in the intrinsic pathway in the present study. The mitochondria-mediated intrinsic pathway is dependent on the release of cytochrome $c$, which leads to the caspase-9-dependent activation of caspase-3 (31). Conversely, 
the death receptor-induced extrinsic pathway signals in a caspase-8 dependent manner (31). The overexpression of TSLNC8 promoted, whereas knockdown of TSLNC8 inhibited the activities of caspase- 3 and caspase-9, but not caspase- 8 in the present study. However, the detailed mechanisms of the promoting effects of TSLNC8 on cell apoptosis in human glioma require further investigation in the future.

In conclusion, the present study revealed the importance of a novel lncRNA, TSLNC8, in human glioma. TSLNC8 was demonstrated to inhibit cell proliferation and metastasis, and promote cell apoptosis in glioma cells. These findings indicated that TSLNC8 may serve as a potential therapeutic target for the diagnosis and treatment of glioma in clinic.

\section{Acknowledgements}

Not applicable.

\section{Funding}

Not applicable.

\section{Availability of data and materials}

The analyzed data sets generated during the study are available from the corresponding author on reasonable request.

\section{Authors' contributions}

DC performed the experiments. XY designed the study, analyzed the data and wrote the manuscript.

\section{Ethics approval and consent to participate}

The present study was approved by the Ethics Committee of Tianjin First Center Hospital. Each patient provided informed consent to participate.

\section{Patient consent for publication}

Written informed consent was obtained.

\section{Competing interests}

The authors declare that they have no competing interests.

\section{References}

1. Taylor LP: Diagnosis, treatment, and prognosis of glioma: Five new things. Neurology 75 (18 Suppl 1): S28-S32, 2010.

2. Gourlay J, Morokoff AP, Luwor RB, Zhu HJ, Kaye AH and Stylli SS: The emergent role of exosomes in glioma. J Clin Neurosci 35: 13-23, 2017.

3. Erdem-Eraslan L, Gravendeel LA, de Rooi J, Eilers PH, Idbaih A, Spliet WG, den Dunnen WF, Teepen JL, Wesseling P, Sillevis Smitt PA, et al: Intrinsic molecular subtypes of glioma are prognostic and predict benefit from adjuvant procarbazine, lomustine, and vincristine chemotherapy in combination with other prognostic factors in anaplastic oligodendroglial brain tumors: A report from EORTC study 26951. J Clin Oncol 31: 328-336, 2013.
4. Ostrom QT, Gittleman H, Farah P, Ondracek A, Chen Y, Wolinsky Y, Stroup NE, Kruchko C and Barnholtz-Sloan JS: CBTRUS statistical report: Primary brain and central nervous system tumors diagnosed in the United States in 2006-2010. Neuro Oncol 15 (Suppl 2): ii1-ii56, 2013.

5. Sukumari-Ramesh S, Prasad N, Alleyne CH, Vender JR and Dhandapani KM: Overexpression of Nrf2 attenuates Carmustine-induced cytotoxicity in U87MG human glioma cells. BMC Cancer 15: 118, 2015

6. Hu B, Emdad L, Bacolod MD, Kegelman TP, Shen XN, Alzubi MA, Das SK, Sarkar D and Fisher PB: Astrocyte elevated gene-1 interacts with Akt isoform 2 to control glioma growth, survival, and pathogenesis. Cancer Res 74: 7321-7332, 2014.

7. Steeg PS, Camphausen KA and Smith QR: Brain metastases as preventive and therapeutic targets. Nat Rev Cancer 11: 352-363, 2011.

8. Hegi ME, Diserens AC, Gorlia T, Hamou MF, de Tribolet N, Weller M, Kros JM, Hainfellner JA, Mason W, Mariani L, et al: MGMT gene silencing and benefit from temozolomide in glioblastoma. N Engl J Med 352: 997-1003, 2005.

9. Reardon DA, Rich JN, Friedman HS and Bigner DD: Recent advances in the treatment of malignant astrocytoma. J Clin Oncol 24: 1253-1265, 2006

10. Spizzo R, Almeida MI, Colombatti A and Calin GA: Long non-coding RNAs and cancer: A new frontier of translational research? Oncogene 31: 4577-4587, 2012.

11. Chu C, Spitale RC and Chang HY: Technologies to probe functions and mechanisms of long noncoding RNAs. Nat Struct Mol Biol 22: 29-35, 2015.

12. Misawa A, Takayama KI and Inoue S: Long non-coding RNAs and prostate cancer. Cancer Sci 108: 2107-2114, 2017.

13. Li J, Meng H, Bai Y and Wang K: Regulation of lncRNA and its role in cancer metastasis. Oncol Res 23: 205-217, 2016.

14. Ramos AD, Attenello FJ and Lim DA: Uncovering the roles of long noncoding RNAs in neural development and glioma progression. Neurosci Lett 625: 70-79, 2016.

15. Ma B, Gao Z, Lou J, Zhang H, Yuan Z, Wu Q, Li X and Zhang B: Long noncoding RNA MEG3 contributes to cisplatininduced apoptosis via inhibition of autophagy in human glioma cells. Mol Med Rep 16: 2946-2952, 2017.

16. Li H, Li Q, Guo T, He W, Dong C and Wang Y: LncRNA CRNDE triggers inflammation through the TLR3-NF- $\kappa \mathrm{B}-\mathrm{Cytokine}$ signaling pathway. Tumour Biol 39: 1010428317703821, 2017.

17. Zhang J, Li Z, Liu L, Wang Q, Li S, Chen D, Hu Z, Yu T, Ding J, Li J, et al: Long noncoding RNA TSLNC8 is a tumor suppressor that inactivates the interleukin-6/STAT3 signaling pathway. Hepatology 67: 171-187, 2018.

18. Wang $\mathrm{MH}$, Lin CL, Zhang JJ, Weng ZP, Hu T, Xie Q and Zhong XY: Role of PTEN in cholera toxin-induced SWO38 glioma cell differentiation. Mol Med Rep 7: 1912-1918, 2013.

19. Livak KJ and Schmittgen TD: Analysis of relative gene expression data using real-time quantitative PCR and the 2(-Delta Delta C(T)) method. Methods 25: 402-408, 2001.

20. Kerr JF, Wyllie AH and Currie AR: Apoptosis: A basic biological phenomenon with wide-ranging implications in tissue kinetics. Br J Cancer 26: 239-257, 1972.

21. Zimonjic DB and Popescu NC: Role of DLC1 tumor suppressor gene and MYC oncogene in pathogenesis of human hepatocellular carcinoma: Potential prospects for combined targeted therapeutics (review). Int J Oncol 41: 393-406, 2012.

22. He Y and Liu X: The tumor-suppressor gene LZTS1 suppresses hepatocellular carcinoma proliferation by impairing PI3K/Akt pathway. Biomed Pharmacother 76: 141-146, 2015.

23. Ganten TM, Haas TL, Sykora J, Stahl H, Sprick MR, Fas SC, Krueger A, Weigand MA, Grosse-Wilde A, Stremmel W, et al: Enhanced caspase- 8 recruitment to and activation at the DISC is critical for sensitisation of human hepatocellular carcinoma cells to TRAIL-induced apoptosis by chemotherapeutic drugs. Cell Death Differ 11 (Suppl 1): S86-S96, 2004.

24. Islam $\mathrm{T}$ and Resat $\mathrm{H}$ : Quantitative investigation of MDA-MB-231 breast cancer cell motility: Dependence on epidermal growth factor concentration and its gradient. Mol Biosyst 13: 2069-2082, 2017.

25. Geng R, Tan X, Wu J, Pan Z, Yi M, Shi W, Liu R, Yao C, Wang G, Lin J, et al: RNF183 promotes proliferation and metastasis of colorectal cancer cells via activation of NF- $\mathrm{KB}-\mathrm{IL}-8$ axis. Cell Death Dis 8: e2994, 2017.

26. Hanahan D and Weinberg RA: Hallmarks of cancer: The next generation. Cell 144: 646-674, 2011. 
27. Hanahan D and Weinberg RA: The hallmarks of cancer. Cell 100: 57-70, 2000.

28. Chaffer CL and Weinberg RA: A perspective on cancer cell metastasis. Science 331: 1559-1564, 2011.

29. Massagué J: TGFbeta in cancer. Cell 134: 215-230, 2008.

30. Chu IM, Lai WC, Aprelikova O, El Touny LH, Kouros-Mehr H and Green JE: Expression of GATA3 in MDA-MB-231 triple-negative breast cancer cells induces a growth inhibitory response to TGFß. PLoS One 8: e61125, 2013.
31. Weller M and Fontana A: The failure of current immunotherapy for malignant glioma. Tumor-derived TGF-beta, T-cell apoptosis, and the immune privilege of the brain. Brain Res Brain Res Rev 21: 128-151, 1995

c) (i) (3) This work is licensed under a Creative Commons (c) AC NO Attribution-NonCommercial-NoDerivatives 4.0 International (CC BY-NC-ND 4.0) License. 